\title{
Parallel pipelined histogram architecture via c-slow retiming
}

Book or Report Section

Accepted Version

Cadenas Medina, J., Sherratt, S., Huerta, P., Kao, W. C. and Megson, G. M. (2013) Parallel pipelined histogram architecture via c-slow retiming. In: Proceedings of the 2013 IEEE International Conference on Consumer Electronics (ICCE). IEEE, pp. 230-231. ISBN 9781467313612 doi: https://doi.org/10.1109/ICCE.2013.6486871 Available at http://centaur.reading.ac.uk/32267/

It is advisable to refer to the publisher's version if you intend to cite from the work. See Guidance on citing.

To link to this article DOI: http://dx.doi.org/10.1109/ICCE.2013.6486871

Publisher: IEEE

All outputs in CentAUR are protected by Intellectual Property Rights law, including copyright law. Copyright and IPR is retained by the creators or other copyright holders. Terms and conditions for use of this material are defined in the End User Agreement. 


\section{www.reading.ac.uk/centaur}

\section{CentAUR}

Central Archive at the University of Reading

Reading's research outputs online 


\title{
Parallel Pipelined Histogram Architecture Via C-slow Retiming
}

\author{
José O. CADENAS, Member, IEEE, R. Simon SHERRATT, Fellow, IEEE, Pablo HUERTA, \\ Wen-Chung KAO, Senior Member, IEEE, and Graham M. MEGSON
}

\begin{abstract}
A parallel pipelined array of cells suitable for realtime computation of histograms is proposed. The cell architecture builds on previous work to now allow operating on a stream of data at 1 pixel per clock cycle. This new cell is more suitable for interfacing to camera sensors or to microprocessors of 8-bit data buses which are common in consumer digital cameras. Arrays using the new proposed cells are obtained via $\mathrm{C}$-slow retiming techniques and can be clocked at a $65 \%$ faster frequency than previous arrays. This achieves over $80 \%$ of the performance of two-pixel per clock cycle parallel pipelined arrays.
\end{abstract}

\section{INTRODUCTION}

Image analysis based on histograms is abundant and well used in many consumer applications [1]. An array of cells to perform the computation of $m$-bin histograms that takes $k$ pixels per clock cycle offers to gain a speedup factor of $k$. Such a design was proposed [2], but required a sensor or processor supplying four pixels per clock cycle to get a speedup of four. Many embedded microprocessors consist of 8-bit data buses and consequently are able to supply one pixel per clock cycle $[3,4]$. In order to exploit this property, a histogram solution using $\mathrm{C}$-slow retiming to create two sub streams of computation derived from a dataset arriving at one pixel per clock cycle is proposed here.

This paper briefly explains the principle of C-slow retiming and applies C-slow to fully develop the proposed cells in section II before presenting final conclusions. The essential result is that the proposed design provides speed-up while also facilitating easier interfacing to camera sensors or microprocessors compared to other designs.

\section{C-SLOWING RETIMING}

C-slow retiming is a method used to reduce the critical path delay in digital circuits especially when feedback loops exist [4]. Every register in the datapath is replaced by $C$ registers and then all registers are moved around on the critical data paths using a retiming algorithm. C-slow retiming separates the calculation performed in the original datapath into $C$ instances. Fig. 1 shows an excerpt of the datapath of a histogram cell previously presented [2] that includes a feedback path (left), its $\mathrm{C}$-slow version by a $C$ factor of two (center) and after retiming to get a $\mathrm{C}$-slow retimed version (right). A simple example using Fig. 1 illustrates the principle of retiming. For input sequence $u=3,5,4,1$ the left diagram in Fig. 1 produces $r=0,3,8,12,13$; the leading zero reflects the register delay with output $r$ being the running accumulation on input $u$. The diagram on the right of Fig. 1 gives $r=0,0,3$, $5,7,6$ for the same input $u$. The output corresponds to the accumulation as if there were two separate input streams: $u_{0}=$ 3, 4 and $u_{1}=5,1$ and as such the output has been separated into $r_{0}=3,7$ and $r_{1}=5,6$; and the two interleaved into output $r$. In general C-slow retiming creates $C$ interleaved streams of computation and as such also requires $C$ input data streams. For practical reasons related to the design, only the factor $C=$ 2 is considered in the rest of the discussion.
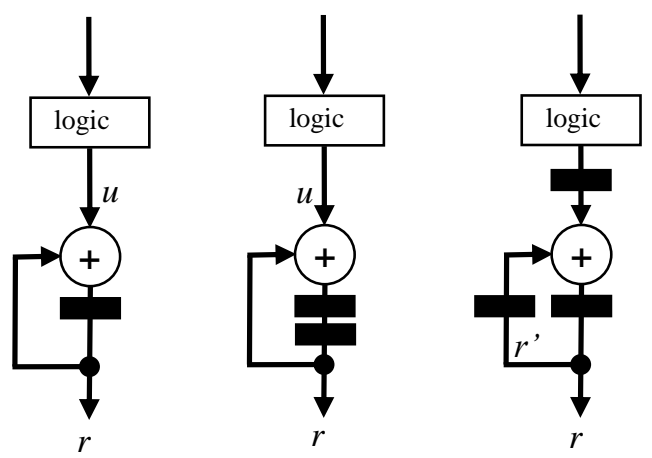

Fig. 1. Pipelined datapath with feedback (left), C-slow with $\mathrm{C}=2$ (center) and C-slow retimed (right).

\section{A. Discussion on the C-slow effects}

Fig. 1 demonstrates that the process of re-timing reduces the critical path delay from the cost of a binary adder and the associated logic to being either the time of the binary adder or the logic time whichever is longer. The downside is that the register count may increase significantly (by a factor of $C$ ). For example, compare the diagrams in Fig. 1 as retiming proceeds from left to right. The final architecture is also influenced by the specific places within the datapath where the registers are finally placed (due to datapath widths.) So, $r^{\prime}=0,0,0,3,5,7$ (Fig. 1 right) and $r+r^{\prime}=0,0,3,8,12,13$ implies the cost of an extra adder is required to merge the two streams; this is unavoidable in the context of the example and also applied to computation of histograms.

\section{B. C-slow retimed histogram processing cell}

A C-slow retimed $(C=2)$ processing cell for the computation of histograms is presented in Fig. 2. This follows straightforwardly from the above discussion and the histogram cell presented [2]. The new registers introduced by C-slow retiming are shown in gray. The mechanism to read bins out from the cell in a pipelined fashion has been omitted for simplicity.

The cell structure above the Logic block has been preserved except for the fact that $\mathrm{C}$-slowing by a factor of two replicates the pipeline registers moving data left to right in the original 


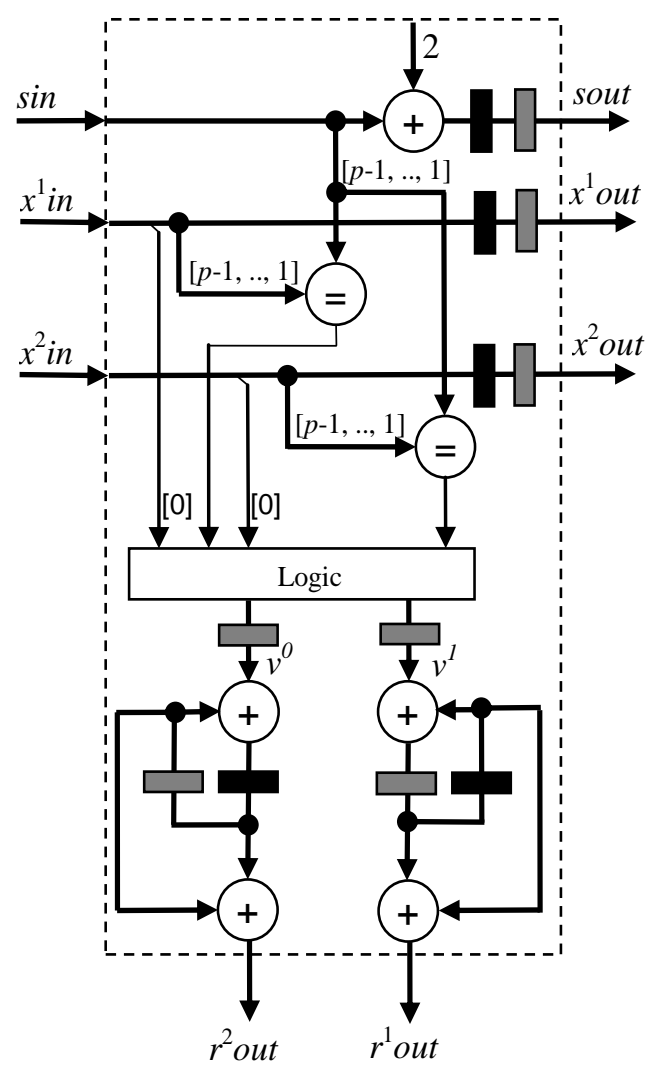

Fig. 2. C-slow retimed cell internal structure processing two data items while computing two histogram bins.

design. It should be appreciated that the structure looks very much like an instance of Fig. 1. It follows that, the separation of the computation into two streams does require the use of the extra adders as seen at the very bottom of Fig. 2. The critical path delay for the cell is now either the comparison followed by the block of Logic or the adder. Without C-slow retiming the critical path is due to the compare-logic-accumulation chain.

\section{Results and analysis}

A design was tested using ASIC technology of 35 microns giving the results in Table 1. Although the C-slow cell is only around 25\% faster than the standard pipelined cell [2] the real advantage comes when the cells are arranged as an array. A pipelined array accepting 2 data items per clock cycle computes the histogram in $n / 2+m / 2$ clock cycles with each cell processing two bins; $m / 2$ is the latency. The C-slow cell in Fig. 2 requires two data items per clock cycle. Assume the cell of Fig. 2 is fed with every other data item (from an input dataset of $n$ items) every clock cycle: half the items go into the array stream piped through $x^{1}$ in input and the other half into through $x^{2}$ in input. As a result an array processes a single data item per clock cycle. Thus, the histogram is computed in $n+m$ clock cycles (the latency is $m$ even for cells computing two bins since each cell in Fig. 2 has a latency of two clock cycles.) As $n \gg m$ for typical image sizes, latency can be ignored for a quick analysis.
TABLE I

HISTOGRAM ARRAY FREQUENCY AND AREA

\begin{tabular}{lcc}
\hline \hline & MHz & No. gates \\
\hline Cell [2] & 226 & 562 \\
C-slow retimed Fig. 2 & 282 & 1366 \\
Histogram array [2] & 144 & 86336 \\
Histogram array of C-slow cells of Fig. 2 & 238 & 194840 \\
\hline
\end{tabular}

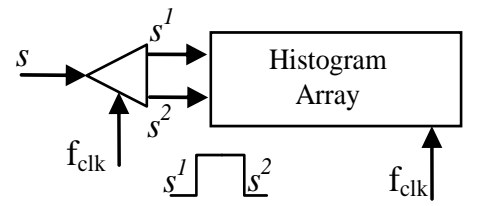

Fig. 3. Principle of operation of a double data rate to create two input streams out of a single input stream.

Arrays of C-slow retimed cells can be clocked $65 \%$ faster than the histogram arrays previously proposed [2]. In fact, from Table 1, ratio $T_{\text {pipe }} / T_{\text {C-slow }}=1.65$ between the pipelined array and the $\mathrm{C}$-slow array, then the time to compute the histogram for any dataset of size $n$ with the C-slow array (one data item per clock cycle) reaches over $80 \%$ of the throughput delivered by a parallel (of two data items per clock cycle) pipelined array. The separation into two streams from a single dataset can be accomplished using a double data rate arrangement [5]. The principle of operation of dual data rate is shown in Fig. 3. The single stream $s$ is distributed into two sub streams $s^{1}$ and $s^{2}$ by a de-multiplexer operating at both edges of the clock, so streams $s^{1}$ and $s^{2}$ are both output at a frequency $f_{\text {clk }}$.

\section{CONCLUSIONS}

A new array of cells computes $m$-bins histograms on streams of one pixel per clock cycle at over $80 \%$ of the performance of a pipelined array, working on streams of two pixels per clock cycle. This is due to arrays of C-slow cells achieving 65\% faster clocks than previous pipelined arrays. The proposed array is consequently better suited for when camera sensors or microprocessors are limited to supply one pixel per clock cycle.

\section{REFERENCES}

[1] H.-C. Huang, F.-C. Chang and W.-C. Fang, "Reversible data hiding with histogram-based difference expansion for QR code applications," IEEE Trans. Consumer Electron., vol. 57, no. 2, pp. 779-787, 2011.

[2] J. O. Cadenas, R. S. Sherratt, P. Huerta and W. C. Kao, "Parallel pipelined arrays for real-time histogram computation in consumer devices," IEEE Trans. Consumer Electron., vol. 57, no. 4, pp. 1460$1464,2011$.

[3] K. Yoon, C. Kim, B. Lee and D. Lee, "Single-chip CMOS image sensor for mobile applications," IEEE J. on Solid State Circuits, vol. 37, no. 12, pp. 1839-1845, 2002.

[4] Available: www.mipi.org/specifications/camera-interface\#CPI

[5] C. Leiserson, F. Rose and J. Saxe, "Optimizing synchronous circuits by retiming," $3^{\text {rd }}$ Caltech Conf. on VLSI, 1993.

[6] R. S. Sherratt and Oswaldo Cadenas, "A double data rate architecture for OFDM based wireless consumer devices," IEEE Trans. Consumer Electron., vol. 56, no. 1, pp. 23-26, 2010. 